\title{
Immediate Implant Dentistry and the Selection of Biomaterial to Fill the Gap: Use of Xenografts with Incorporated Atelo-Collagen
}

\author{
Ferdinando D'Avenia ${ }^{1,2}$ and Richard Miron ${ }^{3 *}$ \\ ${ }^{1}$ University Hospital of Parma, Parma, Italy \\ ${ }^{2}$ Clinica Dentale D’Avenia, Private Practice, Parma, Italy \\ ${ }^{3}$ Department of Periodontology, University of Bern, Bern, Switzerland
}

*Corresponding author: Richard Miron, Department of Periodontology, University of Bern, Bern, Switzerland, Tel: (954) 812-5061; E-mail: richard.miron@zmk.unibe.ch

Received date: July 09, 2018; Accepted date: July 23, 2018; Published date: August 02, 2018

Copyright: (C) 2018 D'Avenia F, et al. This is an open-access article distributed under the terms of the creative Commons Attribution License, which permits unrestricted use, distribution and reproduction in any medium, provided the original author and source are credited.

Citation: D'Avenia F, Miron R. Immediate Implant Dentistry and the Selection of Biomaterial to Fill the Gap: Use of Xenografts with Incorporated Atelo-Collagen. Periodon Prosthodon. 2018, Vol.4 No.2:08.

\section{Abstract}

Immediate implant dentistry has become an effective treatment modality for many clinicians to shorten treatment times. Naturally, while these implants are placed more lingually/palatally and a gap is created between the buccal bone and implant surface, an oftenoverlooked parameter has been the choice of biomaterial/bone graft utilized to fill this void. In the present case study, the introduction of an atelo-collagen based bone grafting material is presented with superior biocompatibility owing to the reduction in immunogenic properties of animal-based collagen. Since xenograft bone biomaterials are commonly devoid of all collagen and growth factor content (reducing their potential for causing an immune response), the recent development of a natural bovine bone mineral containing atelo-collagen type I has favoured atelo-collagen-incorporation within the bone matrix of xenografts. In the present case report, a vertical root fracture on a previously endodonticallytreated right upper first premolar was extracted and an implant was immediately placed. The gap was then packed with an atelo-collagenized xenograft bone graft. A 20-month healing period is provided. This article aims to provide the clinician with a better understanding of the processing steps required to turn collagen into atelocollagen via atelopeptidation and Iyophilization technologies and results in a modification to the immunecollagen component of collagen to non-immunogenic atelo-collagen.

Keywords: Bone graft, Immediate implant dentistry, Atelo-collagen, Osteogenesis, Bone regeneration

\section{Introduction}

The placement of immediate implants into fresh extraction sockets has become routine for many surgically-based clinicians aiming to speed treatment modalities. While once considered 'risky', today it is well known that immediate implant dentistry can achieve similar survival rates as those described using a delayed approach $[1,2]$. One topic that has been debated in recent years has been the choice of bone grafting material utilized to fill the gap created between the remaining buccal wall and the implant surface. Many studies (both pre-clinical and clinical with/without implants) have found marked dimensional alterations occurring post extraction if no biomaterial is utilized [3-5].

A few concepts have been proposed to influence dimensional change post-extraction. First the presence of a thin buccal wall, often characterized as less than $1 \mathrm{~mm}$, is more prone to resorption $[3,4,6,7]$. This remains prominent especially in the esthetic zone where the buccal plate is often thinner than $1 \mathrm{~mm}[6,7]$. If a proper selection criterion is not enforced, there is an increased risk of implant exposure to the midfacial implant surface from mucosal recession, which in certain clinical studies has been reported to occur as high as $40 \%$ of the time in immediate implant dentistry cases [8-10]. Plausible factors that may be responsible for these unsatisfactory esthetic outcomes include 1) facial bone wall thickness, 2) tissue biotype, 3) implant type, 4) implant size and 5 ) implant positioning $[11,12]$

Today it is well known that a proper surgical technique is of primary importance for the successful placement of immediate implants. Nevertheless, also essential is the choice of biomaterial utilized to minimize dimensional changes that occur post-extraction and to maximize bone formation resulting in more favourable clinical and esthetic results. Much research to date has focused on the bone-inducing ability of certain classes of bone grafting materials [13,14]. Nevertheless, very little investigation thus far has investigated 
the possibility to further improve/optimize the regenerative potential of each of these classes of biomaterials.

Xenografts are a class of bone grafting material highly utilized in dentistry owing to their low substitution rate/ properties. The most commonly utilized xenografts are completely devoid of proteins with growth factors removed during thermal processing of the grafting material for sterilization purposes [14]. The end-result is a deproteinized graft that maintains its mineralized properties. Nevertheless, xenografts are one of the most widely used biomaterials in dentistry [15-17].

Recently, the fabrication and processing of xenografts have made major advancements. Sterilization procedures have been modified utilizing atelopeptidation and lyophilization technologies that transform the immune-collagen components of collagen to non-immunogenic atelo-collagen $[18,19]$. It has been found that processing of xenografts utilizing these technologies preserves the natural properties of collagen with an end-product containing roughly $30 \%$ collagen type I.

It has therefore been hypothesized that the regenerative potential of these atelo-collagen-derived bone grafts may potentially further promote new bone formation. In the present case report, we demonstrate how the use of atelocollagen derived bone grafts and a barrier membrane were utilized in an immediate implant case to fill the implant gap. We demonstrate a case follow up time of 20 months with minimal dimensional change or bone loss that occurred over this 20-month period.

\section{Case Report}

A 48-year-old female patient presents to the D'Avenia Dental Clinic in Parma, Italy with complaint of pain on an upper right first pre-molar. Figure 1 demonstrates the clinical presentation of the case. A subsequent $\mathrm{X}$-ray and clinical investigation revealed a vertical root fracture on a previously endodontically-treated right upper first premolar (Tooth 1.4, (Figure 2)). The tooth was deemed non-restorable owing to the extent of the fracture. The patient was presented several options including replacement with a dental implant. Following discussion, the patient elected to have an immediate implant placed with immediate temporization. The case demonstrated adequate buccal bone thickness $(>1 \mathrm{~mm})$ with a thick tissue biotype.

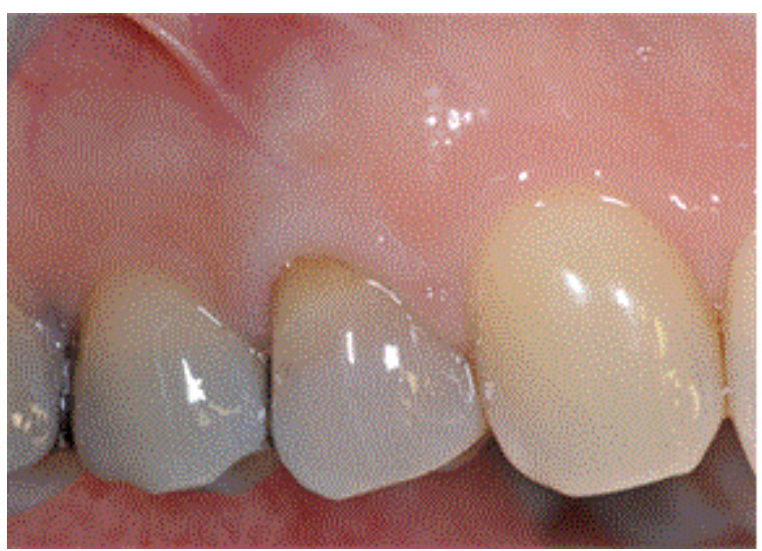

Figure 1 Clinical photo demonstrating tooth 1.4.



Figure 2 Patient presents with a vertical fracture on a previously treated endodontic tooth 1.4 .

Figure 3 depicts the digital planning of the implant case. As can be observed, the implant was planned more palatally than the original tooth in order to maintain the buccal bone and create a space to pack a biomaterial into the gap. The gap was filled with a xenograft that is derived from a bovine source that integrates atelo-collagen within its matrix (ImploBone particle sizes ranging from 0.5 to $1 \mathrm{~mm}$, Bioimplon, Germany, (Figure 4)). Figure 5 illustrates a series of clinical images depicting the clinical steps utilized in this case report as follows: 1) atraumatic tooth extraction, 2) immediate implant placement with a $4.3 \times 13 \mathrm{~mm}$. MIS V3 implant (M.I.S. Savion, Israel), 3) packing of the gap with an atelo-collagenized bone xenograft, 4) temporization of the crown, and 5) final preparation of the temporary crown. Figures 6 and 7 demonstrate the vertical root fracture from both the mesial and distal aspects respectively. Figure 8 illustrates the baseline photo of the final provisional restoration on the day of tooth extraction/immediate implant placement. The height of the buccal cusp was reduced, and the occlusion was slightly more lingualized redistribute and minimizes forces was considered. 


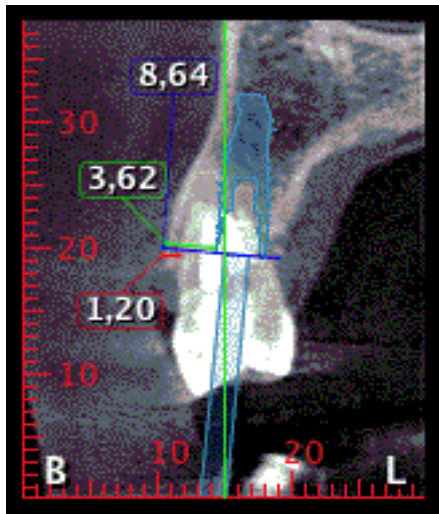

Figure 3 Digital implant planning with ideal implant positioning. The labial surface of the buccal bone demonstrated a $1.22 \mathrm{~mm}$ thickness - adequate for immediate implant placement.

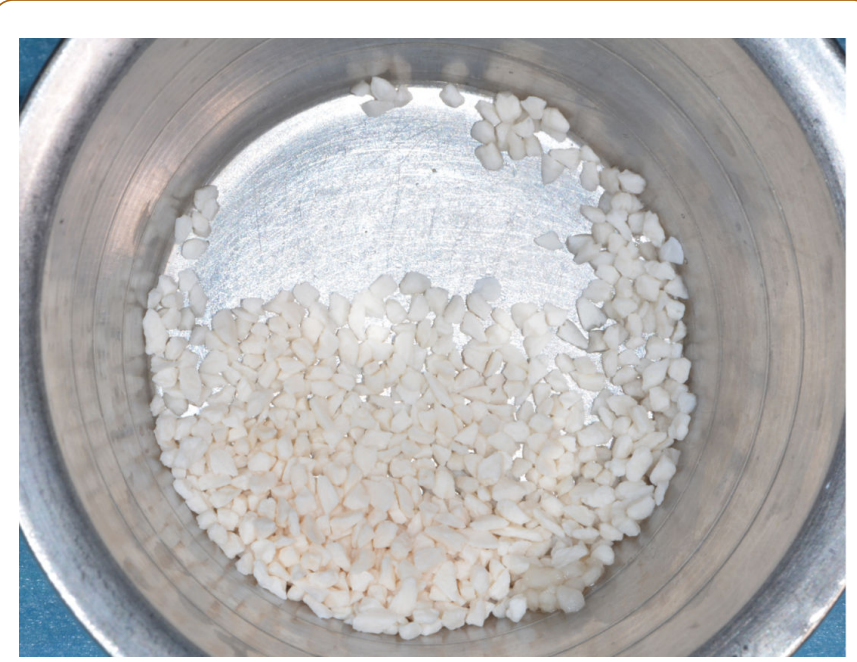

Figure 4 Clinical appearance of atelo-collagenized bone grafting xenograft (ImploBone, Biolmplon).

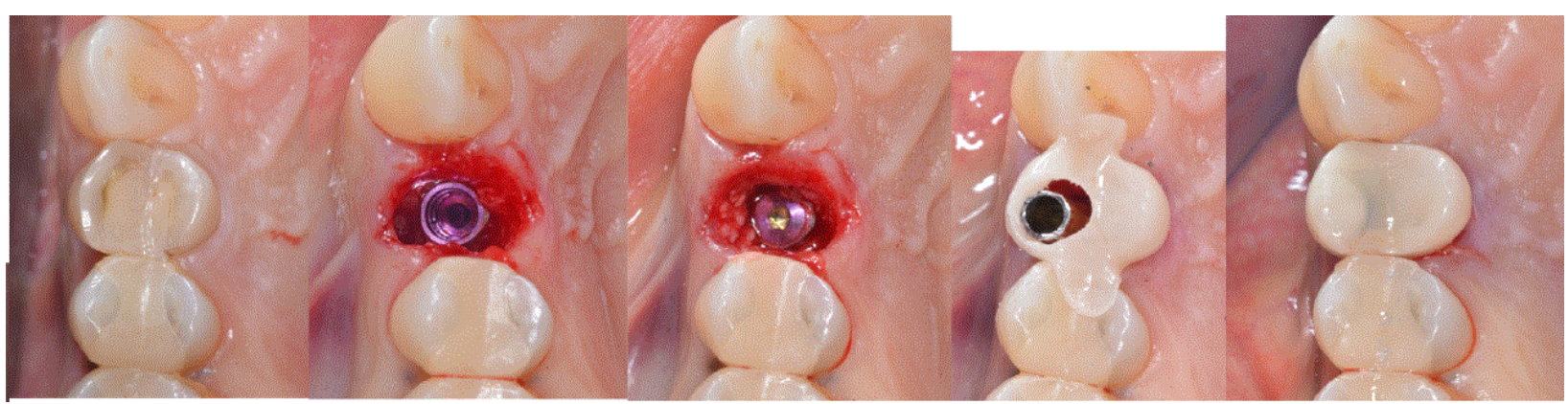

Figure 5 Case demonstration. Initial tooth that was atraumatically extracted. Implant placement. The gap was then packed with ImploBone $(0.2-1 \mathrm{~mm}$, Bioimplon). A provisional restoration was then fabricated and finalized. 


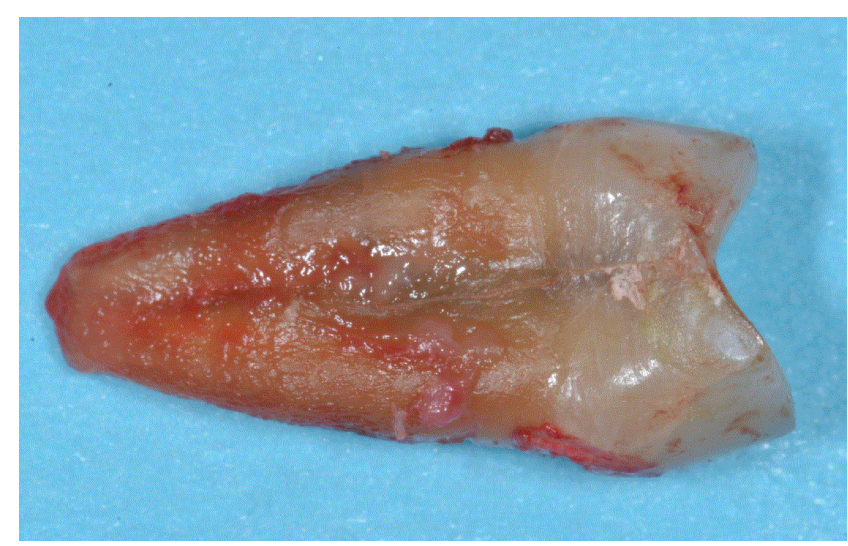

Figure 6 Mesial root fracture - notice the fracture half way down the tooth root.

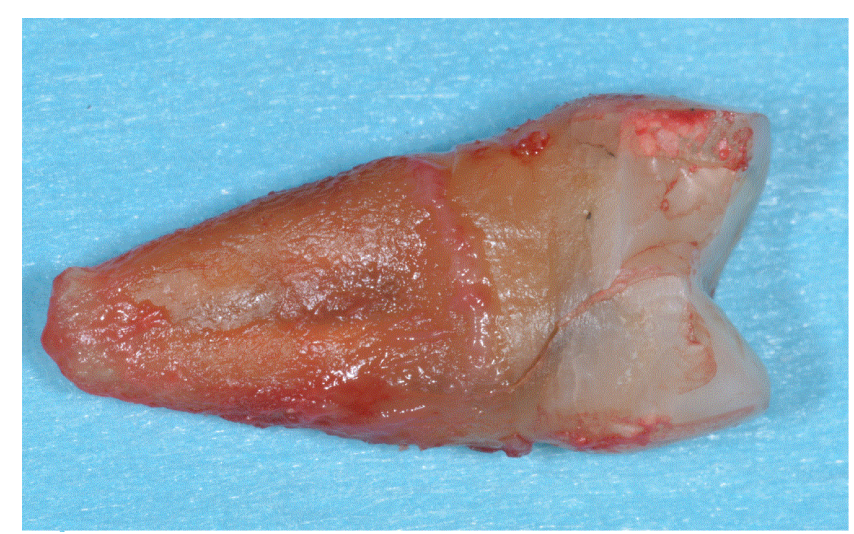

Figure 7 Distal root fracture - notice the fracture that extends lingually.

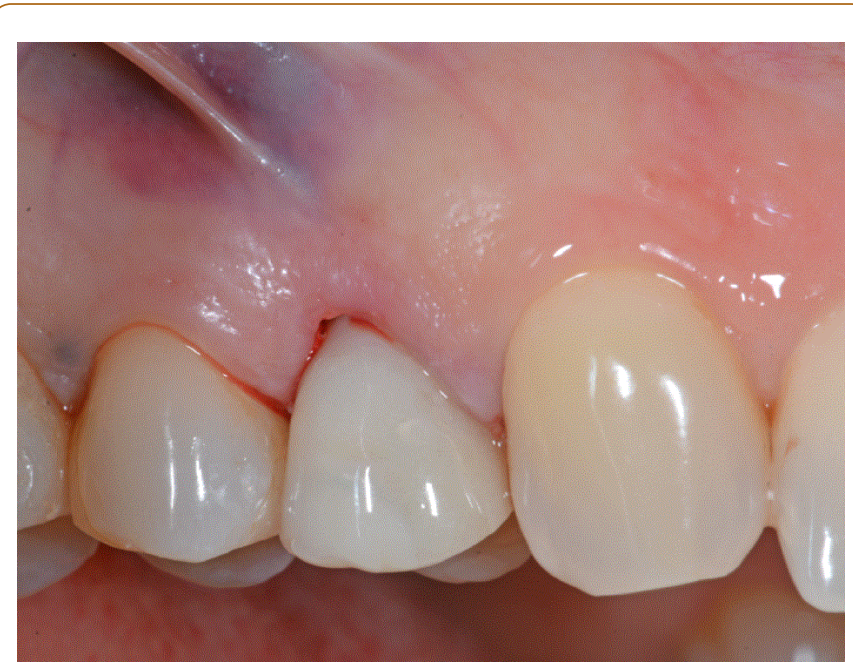

Figure 8 Baseline photo of the provisional restoration immediately following immediate implant placement.

Figure 9 demonstrates the clinical and X-ray photos at 3 months. Notice the excellent soft tissue adaptation and the bone levels around the implant. Figure $\mathbf{1 0}$ illustrates the clinical and X-ray photos at 4 months when the final impression was taken. Figure $\mathbf{1 1}$ demonstrates the final restoration restored at 5 months. Notice the excellent soft tissue adaptation (Figure 12). Figures 13 and $\mathbf{1 4}$ demonstrate the long-term stability of this case utilizing these biomaterials. First, notice the excellent soft tissue maintenance over a 20 month period (Figure 13). Second, notice the implant bone shoulders on both the mesial and distal aspects of the implant (Figure 14). Figure 15 demonstrates the before and after width of the tooth and implant. Originally, an $8.64 \mathrm{~mm}$ distance was measured. After 20 months, the buccal-palatal width of the implant remained stable at $8.41 \mathrm{~mm}$. Furthermore, $3.54 \mathrm{~mm}$ of bone remains present on the buccal surface of the implant, wide enough to favor bone maintenance over time (Figure 15).


Figure 9 Clinical photograph and X-ray at 3 months post-op. Notice the excellent soft tissue wound healing. 

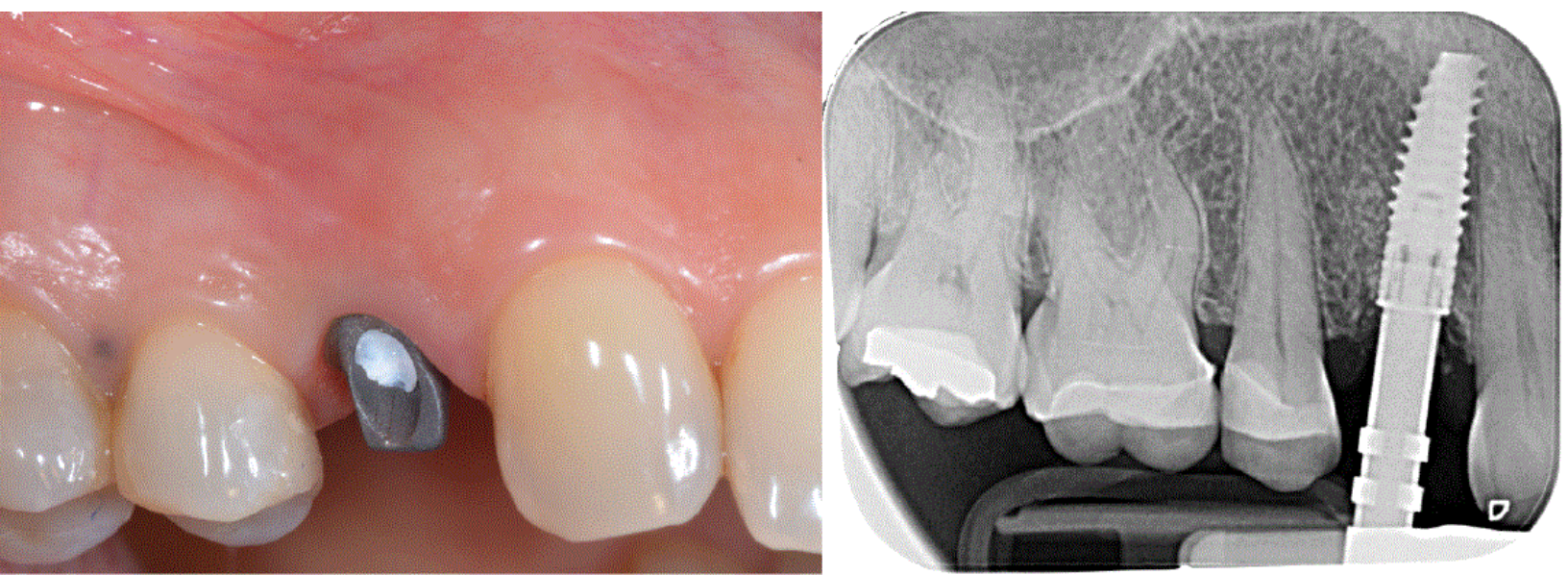

Figure 10 Final impression at 4 months post-op. Once again notice the excellent soft tissue healing and maintenance of papilla on both the mesial and distal sites of the implant.



Figure 11 Final restoration at 5 months post-op.

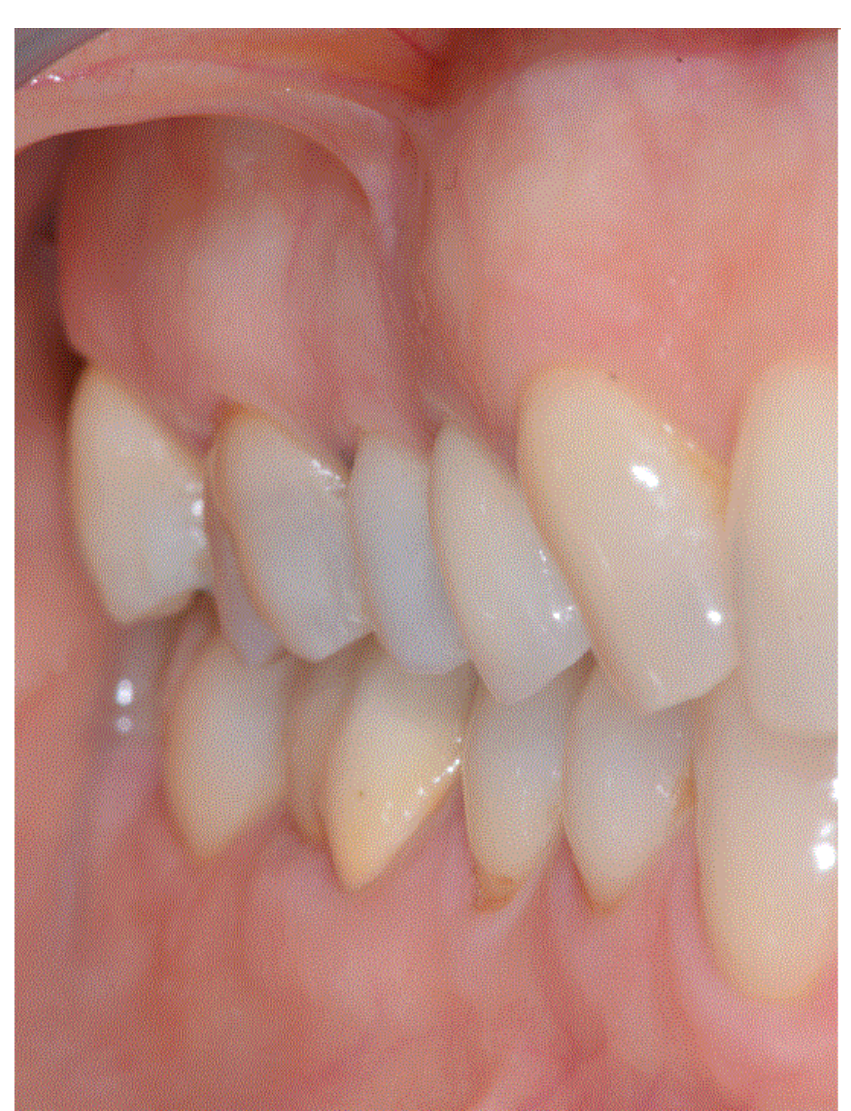

Figure 12 Final restoration at 5 months post-op. 


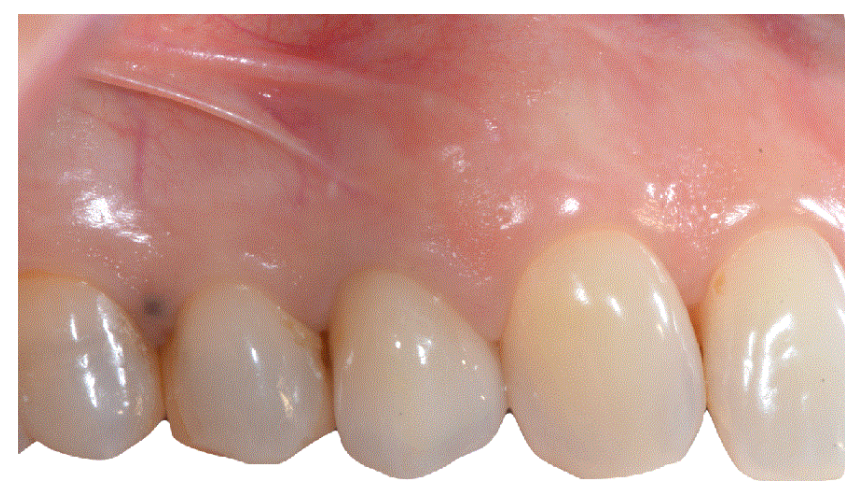

Figure 13 Final restoration at 20 months post-op.

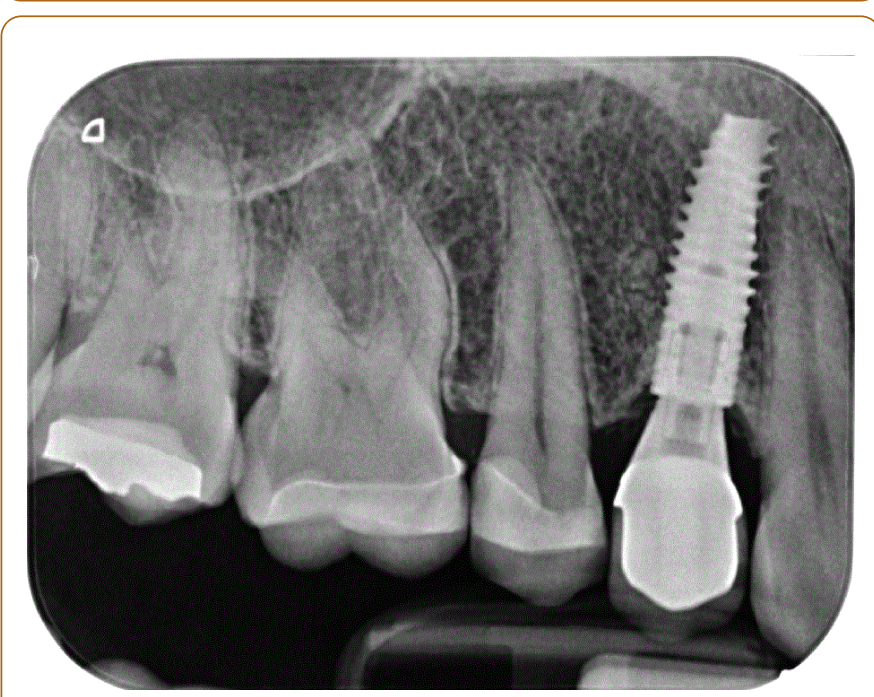

Figure 14 Final X-ray at 20 months post-op. Notice the excellent long-term maintenance of bone around the implant and inter-proximally.
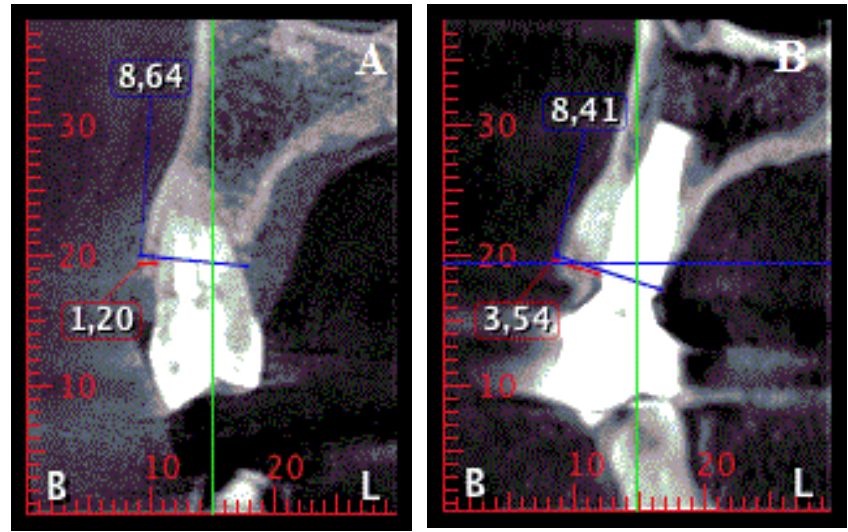

Figure 15 Pre and 20-month post-op CBCT images. Notice the excellent maintenance of the bone dimension over time. Originally measured at $8.64 \mathrm{~mm}$ in width. 20 months later the dimension remains at $8.41 \mathrm{~mm}$. Furthermore, while $1.2 \mathrm{~mm}$ of buccal bone was present pre-op, the finding from 20 months post-op revealed a 3.54 buccal bone surface.

\section{Discussion}

The present case report demonstrates the successful use of an immediate implant that was placed slightly palatally with the 'gap' being filled with an atelo-collagen derived xenograft. As the year's progress, there has been increasing interest to shorten treatment times by placing implants immediately into fresh sockets. While clinically this requires a more advanced surgical skillset, the desired end-goal of immediate implant placement is to better optimize gingival contous yet maintain the dimensional width prior to tooth loss [20].

The clinical question/concern that is often expressed is the alterations in hard tissues that occur post extraction [5,21]. When the tooth is extracted, as is the periodontal ligament that contains a bundle of blood vessels typically responsible for supply blood flow to the surrounding bone. This has been particularly prominent when the buccal bone plate is less than $1 \mathrm{~mm}$ in thickness [6,7]. In present case report, immediate implant placement was considered based on two primary indications: 1) the remaining buccal plate was greater than 1 $\mathrm{mm}$ in size and 2) the patient presented with a thick biotype.

Today it is well known that the positioning of implants during placement, should be more palatally. During immediate implant placement, this creates the so called buccal 'gap' between the exposed implant surface and the buccal bone. This gap may then be filled with a variety of biomaterials capable of inducing bone formation. In the present study, we hypothesized that the use of a xenograft containing atelocollagen would better serve the function of promoting more ideal bone regeneration. It was recently shown that the inclusion of atelo-collagen within the xenograft offers numerous advantages when compared to xenografts devoid of collagen [19]. Atelo-collagen has been shown to favour better 
growth factor adsorption, improve cellular attachment, promote faster cell proliferation and leads to greater osteoblast differentiation [19].

The processing technology was described in a recent study. During the processing of typically xenografts, heat (thermal) sterilization is typically utilized for sterilization purposes however the increasing temperatures have also been associated with both destroying the remaining protein content from the bone graft as well as negatively impacting the natural crystalline micro-structure of hydroxyapatite. The advanced sterilization procedures found in atelo-collagen-derived xenografts preserves the lyophilized collagen which also favours the hydrophilicity of the bone graft. In total, it has been shown that these grafts contain roughly $2 \%$ moisture, $65-75 \%$ hydroxyapatite, $25-35 \%$ atelo-collagen and up to $0.1 \%$ non-collagenous proteins [19]. We therefore were interested to utilize this technology in an immediate implant grafting case. To the best of both the authors knowledge, this is the first case report investigating the use of atelo-collagen based xenografts for such indications.

While the surgical procedure was as planned, most notably, the authors report the excellent soft tissue healing. It may therefore be reported that the superior biocompatibility of atelo-collagen over collagen is thought to have contributed to the final results by minimizing tissue inflammation and speed wound healing. Second, the buccal-palatal distance in the present case report was well maintained even at 20 months post-op. While this case will certainly be followed over time to further document changes at 5+ years, these initial healing results are very encouraging. Further histological analysis is also of interest to further determine the quality and quantity of bone formation taking place around these grafting particles.

\section{Conclusion}

This case report describes a surgical approach whereby implant placement was significantly shortened owing to the ability to immediately place the implant with immediate temporization. A novel xenograft bone graft that incorporated atelo-collagen within the graft complex was utilized in this study and was shown to favourably promote bone formation, likely owing to the superior biocompatibility of atelo-collagen. Future comparative studies and histological studies are now necessary to further validate this novel biomaterial.

\section{References}

1. Hur $Y$, Ogata $Y(2016)$ No clinically significant differences in crestal bone loss between immediate implant placement and implants placed in healed bone: A systematic review of articles published from 1966 through 2012. J Am Dent Assoc 147: 987-989.

2. Poskevicius L, Sidlauskas A, Moreno GP, Juodzbalys G (2017) Dimensional soft tissue changes following soft tissue grafting in conjunction with implant placement or around present dental implants: a systematic review. Clin Oral Implants Res 28: 1-8.

3. Schropp L, Kostopoulos L, Wenzel A (2003) Bone healing following immediate versus delayed placement of titanium implants into extraction sockets: a prospective clinical study. Int J Oral Maxillofac Implants 18: 189-99.

4. Botticelli D, Persson LG, Lindhe J, Berglundh T (2006) Bone tissue formation adjacent to implants placed in fresh extraction sockets: an experimental study in dogs. Clin Oral Implants Res 17: 351-358.

5. Araújo MG, Lindhe J (2005) Dimensional ridge alterations following tooth extraction. An experimental study in the dog. J Clin Periodont 32: 212-218.

6. Chappuis V, Engel O, Reyes M, Shahim K, Nolte LP, et al. (2013) Ridge alterations post-extraction in the esthetic zone: a 3D analysis with CBCT. J Dent Res 92: 195s-201s.

7. Chappuis V, Engel O, Shahim K, Reyes M, Katsaros C, et al. (2015) Soft Tissue Alterations in Esthetic Postextraction Sites: A 3Dimensional Analysis. J Dental Res 94: 187s-193s.

8. Araujo MG, Sukekava F, Wennstrom JL, Lindhe J (2005) Ridge alterations following implant placement in fresh extraction sockets: an experimental study in the dog. J Clin Periodontol 32: 645-652.

9. Botticelli D, Renzi A, Lindhe J, Berglundh T (2008) Implants in fresh extraction sockets: a prospective 5-year follow-up clinical study. Clin Oral Implants Res 19: 1226-1232.

10. Kan JY, Rungcharassaeng K, Sclar A, Lozada JL (2007) Effects of the facial osseous defect morphology on gingival dynamics after immediate tooth replacement and guided bone regeneration: 1year results. J Oral Maxillofac Surg 65: 13-19.

11. Buser D, Chappuis V, Belser UC, Chen S (2017) Implant placement post extraction in esthetic single tooth sites: when immediate, when early, when late? Periodontol 73: 84-102.

12. Chen ST, Buser D (2014) Esthetic outcomes following immediate and early implant placement in the anterior maxilla--a systematic review. Int J Oral Maxillofac Implants 29: 186-215.

13. Miron RJ, Sculean A, Shuang $Y$, Bosshardt DD, Gruber R, et al. (2016) Osteoinductive potential of a novel biphasic calcium phosphate bone graft in comparison with autographs, xenografts, and DFDBA. Clin Oral Implants Res 27: 668-675.

14. Miron RJ, Zhang Q, Sculean A, Buser D, Pippenger BE, et al. (2016) Osteoinductive potential of 4 commonly employed bone grafts. Clin Oral Invest 20: 2259-2265.

15. Buser D, Chappuis V, Kuchler U, Bornstein MM, Wittneben JG, et al. (2013) Long-term stability of early implant placement with contour augmentation. J Dent Res 92: 176s-182s.

16. Jensen SS, Aaboe M, Janner SF, Saulacic N, Bornstein MM, et al. (2015) Influence of particle size of deproteinized bovine bone mineral on new bone formation and implant stability after simultaneous sinus floor elevation: a histomorphometric study in minipigs. Clin Implant Dent Res 17: 274-285.

17. Jensen SS, Bosshardt DD, Gruber R, Buser D (2014) Long-term stability of contour augmentation in the esthetic zone: histologic and histomorphometric evaluation of 12 human biopsies 14 to 80 months after augmentation. J Periodontol 85: 1549-1556.

18. El Raouf MA, Fujioka-Kobayashi M, AbdEl-Aal ABM, Zhang $Y$, Miron RJ (2017) Novel Bioabsorbable Bovine Derived AteloCollagen Type I Membrane: Characterization into Host Tissues. Periodon Prosthodon 3: 1-6.

19. Fujioka-Kobayashi M, Schaller B, Saulacic N, Zhang Y, Miron RJ (2017) Growth factor delivery of BMP9 using a novel natural bovine bone graft with integrated atelo-collagen type I: 
Biosynthesis, characterization, and cell behavior. J Biomed Mater Res 105: 408-418.

20. Lang NP, Pun L, Lau KY, Li KY, Wong MC (2012) A systematic review on survival and success rates of implants placed immediately into fresh extraction sockets after at least 1 year. Clin Oral Implants Res 5: 39-66.
21. Chappuis V, Araujo MG, Buser D (2017) Clinical relevance of dimensional bone and soft tissue alterations post-extraction in esthetic sites. Periodontol 73: 73-83. 